\title{
Painful Symptomatology Reported by Dentistry Students at a Brazilian University
}

\author{
Cristiane Assunção da Costa Cunha ${ }^{1}$, Iris do Ceu Clara Costa ${ }^{1}$, Angelo Giuseppe Roncalli' \\ Dyego Leandro Bezerra de Souza ${ }^{2}$, Fábia Barbosa de Andrade ${ }^{3}$, Antônio Medeiros Júnior ${ }^{2}$ \\ ${ }^{1}$ Department of Dentistry, Federal University of Rio Grande do Norte (UFRN), Natal, Brazil \\ ${ }^{2}$ Department of Collective Health, Federal University of Rio Grande do Norte (UFRN), Natal, Brazil \\ ${ }^{3}$ Department of Nursing, Faculty of Health Sciences of Trairi (FACISA), Federal University of Rio Grande do \\ Norte (UFRN), Santa Cruz, Brazil \\ Email: cris.acc@hotmail.com, iris odontoufrn@yahoo.com.br, roncalli@terra.com.br, \\ dyegosouz@yahoo.com.br, fabiabarbosabr@yahoo.com.br, amjunior@ufrnet.br
}

Received 1 September 2014; accepted 11 August 2015; published 14 August 2015

Copyright (C) 2015 by authors and Scientific Research Publishing Inc.

This work is licensed under the Creative Commons Attribution International License (CC BY).

http://creativecommons.org/licenses/by/4.0/

(c) (i) Open Access

\begin{abstract}
Objectives: Identification of the level of knowledge on ergonomics principles, and application of these by dentistry students to investigate whether painful symptomatology was experienced. Another objective is the expansion of discussions on occupational health in academic settings. Study Design and Settings: Dentistry students of the Federal University of Rio Grande do Norte, Brazil ( $n=148$ ) were surveyed using the Nordic Musculoskeletal Questionnaire to determine the severity of musculoskeletal symptoms experienced. Data were analyzed through EpiInfo 7.0 to measure central trends and variability 5 for quantitative variables, absolute and relative frequencies for categorical variables, and significance between groups (confidence intervals and chisquare). Association analysis (Pearson) was also carried out. Results: Ergonomic measures were not reported by students. Within the musculoskeletal symptoms described, females were the most affected, independent of academic level. Conclusions: Positive correlations were verified between all categories and all anatomic regions (e.g., neck, lower back, wrists, hands, and shoulders). Data suggest progressive worsening of symptoms, which will eventually result in leaves of absence.
\end{abstract}

\section{Keywords}

Musculoskeletal Disorders, Ergonomics, Health Promotion, Occupational Health, Health Education

\section{Introduction}

Occupational diseases are often triggered by excessively repetitive motions, awkward posture, or workplace fa-

How to cite this paper: da Costa Cunha, C.A., do Ceu Clara Costa, I., Roncalli, A.G., de Souza, D.L.B., de Andrade, F.B. and Medeiros Júnior, A. (2015) Painful Symptomatology Reported by Dentistry Students at a Brazilian University. Health, 7, 976985. http://dx.doi.org/10.4236/health.2015.78115 
tigue. There is no standard terminology for occupational diseases, which are referred to as Repetitive Strain Injury (RSI) in Canada, the United States (USA), and Europe, Occupational Overuse Syndrome in Australia and the Netherlands, complications in the arms, necks, and shoulders in Brazil, lesions attributed to repetitive work in France, and cervicobrachial occupational disorders in Japan [1].

Regardless of the terminology used, illnesses in the workplace often reflect the statistics of an economically active population. In the USA, approximately two billion dollars are spent annually on individuals affected by musculoskeletal disorders in the arms, necks, and shoulders. This corresponds to $56 \%$ - $65 \%$ of all occupational disorders reported [2]. In Brazil, musculoskeletal diseases are part of a wider group of diseases, which include occupational diseases (considered a public health problem [3]). Therefore, research on quality of work life is relevant and very important.

Qualitative questionnaires have been developed to diagnosis and treat occupational morbidity, including questions that address the habits of patients in daily work, history of musculoskeletal diseases, preventive measures for the avoidance of occupational injuries, customer satisfaction, and service quality [4]. Based on the data obtained by these different indices, it is evident that further research is paramount to better identify, interpret, and understand the numerical scores obtained from occupational performance measures. In Canada (COPM), occupational performance measures are derived from various sources, including: questionnaires on quality of life in patients with juvenile arthritis (AQQ), questionnaires regarding arthritis (MACTAR), a Measure Yourself Patient-Specific Index (PASI) for full hip arthroplasty patients, Problem Elicitation Technique (PET) surveys, Patient Generated Index (PGI) surveys of quality of life, Patient-Specific Functional Scale (PSFS) questionnaires, and Schedule for the Evaluation of Individual Quality of Life (SEIQoL) surveys. Data collected from the responses must be evaluated for content, validity, feasibility of use, psychometric properties, and reliability.

A worldwide key challenge in the field of epidemiology is the lack of standardization in the classification of musculoskeletal disorders, particularly regarding the diagnosis and treatment of injuries related to the work environment [5]. Currently, there are 27 classifications of disorders involving muscles, tendons, and nerves that have been reported by different professions. Moreover, in many of the studies, the authors have suggested that a consistent classification of conditions would facilitate an elucidation of the etiology, diagnosis, prevention, and treatment of musculoskeletal disorders [6].

The term RSI itself does not represent a diagnosis. It is utilized to characterize work-related morbidities in different professions. Generally, a patient that describes RSI characteristics is prescribed an intervention, which ranges from physical therapy to the application of ergonomic principles. The latter includes pauses in work time, posture-targeting therapy according to the profession and the affected anatomic region, professional massages, and regular exercise. In addition, discussion of the preventive measures that are relevant to occupational health is important [7].

The prevalence of musculoskeletal complaints in dental professionals has been reported to range from $77 \%$ to $94 \%$. Such a high incidence of repetitive strain injuries is associated with an invariable posture, fast and repetitive motions, extreme flexing or extension of the wrists and fingers, loads on tendons, as well as static mechanical compression of the muscles, fascia, and nerves of the upper and lower limbs, shoulder girdle, neck, and joints [8]-[10]. As a result, approximately $30 \%$ of dental professionals cite musculoskeletal pains as reasons for premature abandonment of the profession. Moreover, $47 \%$ of these morbidities occur after professionals have been practicing for between 10 and 19 years. The most affected age group is between 30 and 49 years of age, and an association has been observed between symptoms of musculoskeletal pain and practicing time when no preventive measures were established [11]-[13].

Research on the relationship between workers and their environment has identified new perspectives regarding the need for balance, wellness, and satisfaction in work relationships. Aspects of the health of workers have been identified, allowing the occupational aspect of several professions to be addressed more comprehensively, with a consideration for paradigms of health promotion and quality of life. In the field of dentistry, technological innovations that have been introduced include newly-engineered materials, the relevance of the genetic background of the patient, aesthetics, prevention of oral diseases, and other numerous accomplishments. However, the stress of daily activities, postural overload of the upper and lower limbs and muscles, excessive noise, and poor planning of equipment location and environment often contribute to the incidence of musculoskeletal diseases and their progression [14].

There is a clear lack of clinical application of ergonomic principles in educational settings. Students undergoing training in the field of dentistry reported painful symptomatology during studies, particularly in the neck, 
lower back, wrists, hands and shoulders. Female students were the majority experiencing these symptoms.

The authors propose an intervention protocol to be added to the institutional biosafety protocol, which includes a presentation of preventive ergonomic practices that can be applied at dental clinics to prevent future illnesses.

The goal of this study is to highlight the role of ergonomics in determining occupational hazards in dentistry, particularly regarding interactions between the individual, their labor, and the equipment and environment involved. According to the International Ergonomics Association (IEA), the field of ergonomics represents a tool, instrument, and science to address issues related to the adaptation of the man to his profession, including the theory, principles, data, and methods that optimize the productivity of a professional as well as longevity in the corresponding field [15] [16]. This work aims at the identification and application of ergonomic principles during an investigation of painful symptomatologies reported by students of dentistry clinics. Another objective of the work herein presented is to expand the discussion on occupational health in academic settings.

Ergonomic considerations refer to those related to adequate work postures, satisfactory levels of workplace lighting, instruments and equipment within healthcare work regulations, utilization of odontology assistants, avoidance of osteomuscular burden, and increase of productivity in an optimized manner, with pauses between patients.

\section{Methods}

\subsection{Population and Study Participants}

The study was conducted at the Federal University of Rio Grande de Norte (UFRN) in the Department of Dentistry, at the Health Sciences Campus. The UFRN University is a medium-sized institution located in Northeast Brazil, with different academic centers in specific fields, including a Center for Health Sciences (CCS). Courses are offered in Nursing, Physiotherapy, Medicine, and Dentistry, to name a few. The dentistry undergraduate course has 80 positions available yearly, and currently counts with a total of 328 students. A full course consists of nine semesters (academic levels), with students between the 5th and 9th semesters participating in different dental clinic courses that assist approximately 1000 patients per year.

For the study herein presented, all students enrolled in clinical courses that corresponded to the 5th, 6th, 7th, 8th, and 9th semester levels of the dentistry courses offered in 2010 participated in the study $(n=148)$. Students that did not participate in dental clinic courses were excluded.

\subsection{Tools and Techniques for Data Collection}

Two data collection instruments were applied: firstly, a survey was conducted during classes of different curriculum courses. The survey included a statement on the objectives of this research and consent terms, and contained questions regarding sociodemographic characteristics, level of knowledge on ergonomics, occupational diseases in dentistry, preventive measures against occupational diseases, and clinical applications of ergonomic guidelines at the university. Surveys were completed during class and included the signature of consent forms.

Secondly, a validated Brazilian version of the Nordic Musculoskeletal Questionnaire was translated into several languages and was handed to students during classes of different curriculum courses. The goal of this survey was to standardize the measurement of reported musculoskeletal symptoms to better identify disorders. The survey included a figure of the human body where the anatomical regions affected (e.g., neck, shoulders, upper and lower back, elbows, wrists, hands, hips, thighs, knees, ankles, and feet) could be selected to represent where symptoms had been experienced.

\subsection{Data Analysis}

An index was created, based on the Nordic questionnaire (Appendix), to represent the severity of symptoms in accordance with Pinheiro et al. (2002) [1] (Table 1) for each anatomic region, with scores ranging from 0 to 4. A score of 0 represented absence of symptoms. Score 1 exposes symptoms reported in the last 7 days or the past 12 months (had pain in a period “or” on the other). The score 2 reports that the person had musculoskeletal symptoms in both periods (had pain in the last seven days "and" also in 12 months). Score 3 considers that the person has had symptoms for seven days or 12 months and in addition had to be absent from work (had symptoms for a period "or" the other "associated with absence" at work) and finally the score 4 reports pain two periods with 
Table 1. Expression of severity of symptoms in students. Natal City, State of the Rio Grande do Norte, Brasil, 2013.

\begin{tabular}{l}
\hline Score "0" \\
Score "1" \\
Score "2" \\
Score "3" \\
Symptoms reported in the previous 12 months or 7 days preceding the interview; \\
Score "4" \\
Symptoms reported in the previous 7 days or preceding 12 months, with leave of absence; \\
\hline
\end{tabular}

Pinheiro FA, Amaral BT, Carvalho CV. Validation of questionnaire Nordic musculoskeletal symptoms as a measure of morbidity. Rev Saúde Pública 2002: 36 (3): 307-312.

absence at work (had pain in both periods, 7 days "and" 12 months "associated with absence"). The 7 days prior to collection can not be compared to the past 12 months because they are different times. In the case of seven days is a very recent memory and the pain may still be bothering you, on the day and time of collection. Instead, a pain that happened 12 months ago, there is a memory bias, he reported that had pain, but may have already forgotten their intensity, how much bothered and interfered in clinical work.

As previously mentioned, a validated Brazilian version of the Nordic Musculoskeletal Questionnaire was also applied. This questionnaire was translated into several languages and was developed with the goal of standardizing measurements of reported musculoskeletal symptoms to identify disorders. It is a diagnostic tool for describing the environment or workplace of an individual, and includes an illustration of the anatomical regions of the human body (e.g., neck, shoulders, upper and lower back, elbows, wrists, hands, hips, thighs, knees, ankles, and feet). This figure allows the respondent to indicate where symptoms have been experienced. The respondent is asked to report the occurrence of symptoms within the past 12 months and 7 days preceding the interview as well as any leave of absence or change in routine activities over the previous year, and to indicate if a health professional has been consulted.

Regarding the statistical procedures, the severity index and the frequency of symptoms were computed as dependent variables. Pearson's correlation coefficient and the corresponding confidence interval were calculated between symptoms in different periods (within the previous 7 days and within the previous 12 months) and leave of absence. The severity index was analyzed through the calculation of means and confidence intervals for each independent variable. For both analyses, the level of confidence was 95\%.

\section{Results}

Table 2 shows that all anatomical regions were correlated when the three presented situations were considered: symptoms presented in the previous 12 months and previous 7 days, symptoms in the last 12 months associated with leave of absence due to pain and lastly, symptoms in the previous 7 days with leave of absence due to pain. Painful symptomatology was reported, highly significant for all anatomical regions $(p<0.001)$, with the highest correlations for elbows, ankles, and feet.

The severity index plus the frequency of symptoms in each region are the dependent variables used in the analysis. Independent variables included student gender and academic level.

As shown in Table 2, positive correlations were observed in all categories (e.g., 12 months $\times$ symptoms within last 7 days, symptoms in 12 months and symptoms with leave of absence $\times 7$ days $\times$ leave of absence) and in all anatomic regions. These results indicate a progressive worsening of symptoms. In addition, if the conditions are not changed, symptoms that occurred throughout one week can continue to be experienced for up to 12 months, and potentially result in exclusion from work activities.

Table 3 presents the means and confidence intervals for the severity index of symptoms presented by students in different anatomical regions, considering age, gender, and academic level of students.

Regarding the academic period in which the students were enrolled, it was observed that, although some significant differences existed (demonstrated by the confidence intervals), there was no dose-answer effect that indicated a possible correlation between the academic age of students and the severity index. The same could be observed for age group and gender, where intervals were superimposed for the majority of analyzed areas.

Figure 1 shows the frequency of symptoms and by consequence, the leave of absence from clinical activities among the students participating in the research. Regarding the presence of pain, it could be verified that in 
Table 2. Pearson's coefficient, " $p$ ” values for different anatomical regions, and leave of absence by students. Natal City, State of the Rio Grande do Norte, Brazil. 2013.

\begin{tabular}{cccc}
\hline Anatomical region & $\begin{array}{c}\text { Symptoms within } \\
\text { previous } 12 \text { months } \times 7 \text { days }\end{array}$ & $\begin{array}{c}\text { Symptoms within previous } \\
12 \text { months } \times \text { leave of absence }\end{array}$ & $\begin{array}{c}\text { Symptoms within previous } \\
7 \text { days } \times \text { leave of absence }\end{array}$ \\
\hline Neck & $0.43(<0.001)$ & $0.41(<0.001)$ & $0.47(<0.001)$ \\
Shoulders & $0.50(<0.001)$ & $0.40(<0.001)$ & $0.59(<0.001)$ \\
Upper back & $0.45(<0.001)$ & $0.44(<0.001)$ & $0.45(<0.001)$ \\
Elbows & $0.69(<0.001)$ & $0.72(<0.001)$ & $0.68(<0.001)$ \\
Fist/hands & $0.46(<0.001)$ & $0.44(<0.001)$ & $0.43(<0.001)$ \\
Lower back & $0.48(<0.001)$ & $0.44(<0.001)$ & $0.41(<0.001)$ \\
Hips/thighs & $0.63(<0.001)$ & $0.64(<0.001)$ & $0.63(<0.001)$ \\
Knees & $0.65(<0.001)$ & $0.62(<0.001)$ & $0.65(<0.001)$ \\
Ankles/feet & $0.71(<0.001)$ & $0.65(<0.001)$ & $0.68(<0.001)$ \\
\hline
\end{tabular}

Table 3. Means and confidence intervals of the severity index for different anatomical regions (dependent variables: age, gender and academic level of students). Natal City, State of the Rio Grande do Norte, Brazil. 2013.

\begin{tabular}{|c|c|c|c|c|c|c|c|c|c|c|c|}
\hline \multirow{2}{*}{ Variable } & \multirow{2}{*}{$\mathrm{n}$} & \multicolumn{2}{|r|}{ Neck } & \multicolumn{2}{|c|}{ Shoulders } & \multicolumn{2}{|c|}{ Upper back } & \multicolumn{2}{|c|}{ Lower back } & \multicolumn{2}{|c|}{ Fist and hands } \\
\hline & & Mean & CI (95\%) & Mean & CI (95\%) & Mean & CI (95\%) & Mean & CI (95\%) & Mean & CI (95\%) \\
\hline \multicolumn{12}{|l|}{ Semester } \\
\hline $5^{\circ}$ & 32 & 1.56 & $1.26-1.86$ & 1.06 & $0.58-1.54$ & 1.25 & $0.84-1.66$ & 1.59 & $1.06-2.12$ & 0.97 & $0.53-1.41$ \\
\hline $6^{\circ}$ & 36 & 0.92 & $0.63-1.21$ & 0.72 & $0.41-1.03$ & 1.03 & $0.64-1.42$ & 0.89 & $0.46-1.32$ & 0.72 & $0.41-1.03$ \\
\hline $7^{\circ}$ & 23 & 0.78 & $0.37-1.19$ & 0.70 & $0.36-1.04$ & 0.91 & $0.52-1.30$ & 0.74 & $0.29-1.19$ & 0.61 & $0.23-0.99$ \\
\hline $8^{\circ}$ & 24 & 1.21 & $0.80-1.62$ & 0.71 & $0.31-1.11$ & 1.17 & $0.73-1.61$ & 1.04 & $0.64-1.44$ & 0.58 & $0.19-0.97$ \\
\hline $9^{\circ}$ & 33 & 1.21 & $0.79-1.63$ & 0.88 & $0.45-1.31$ & 0.94 & $0.54-1.34$ & 1.12 & $0.66-1.58$ & 1.19 & $0.23-1.05$ \\
\hline \multicolumn{12}{|l|}{ Age } \\
\hline$<20$ years of age & 15 & 1.47 & $0.84-2.10$ & 0.67 & $0.08-1.26$ & 0.73 & $0.37-1.09$ & 1.00 & $0.34-1.66$ & 0.80 & $0.32-1.28$ \\
\hline 20 to 30 years of age & 133 & 1.11 & $0.94-1.28$ & 0.84 & $0.65-1.03$ & 1.10 & $0.90-1.30$ & 1.11 & $0.89-1.33$ & 0.71 & $0.52-0.90$ \\
\hline \multicolumn{12}{|l|}{ Gender } \\
\hline Female & 84 & 1.37 & $1.15-1.59$ & 0.93 & $0.69-1.17$ & 1.18 & $0.94-1.42$ & 1.13 & $0.86-1.40$ & 0.80 & $0.57-1.03$ \\
\hline Male & 64 & 0.86 & $0.62-1.10$ & 0.69 & $0.43-0.95$ & 0.91 & $0.63-1.19$ & 1.05 & $0.72-1.38$ & 0.61 & $0.34-0.88$ \\
\hline \multirow{2}{*}{ Variable } & \multirow{2}{*}{$\mathrm{n}$} & \multicolumn{2}{|c|}{ Elbows } & \multicolumn{2}{|c|}{ Hips and thighs } & \multicolumn{2}{|r|}{ Knees } & \multicolumn{2}{|c|}{ Ankles and feet } & & \\
\hline & & Mean & CI (95\%) & Mean & CI (95\%) & Mean & CI (95\%) & Mean & CI (95\%) & & \\
\hline \multicolumn{12}{|l|}{ Semester } \\
\hline $5^{\circ}$ & 32 & 0.06 & $0.00-0.18$ & 0.38 & $0.03-0.73$ & 0.31 & $0.03-0.59$ & 0.25 & $0.05-0.45$ & & \\
\hline $6^{\circ}$ & 36 & 0.22 & $0.03-0.41$ & 0.22 & $0.06-0.38$ & 0.28 & $0.05-0.51$ & 0.28 & $0.01-0.55$ & & \\
\hline $7^{\circ}$ & 23 & 0.09 & $0.00-0.21$ & 0.17 & $0.00-0.37$ & 0.39 & $0.07-0.71$ & 0.30 & $0.07-0.53$ & & \\
\hline $8^{\circ}$ & 24 & 0.29 & $0.00-0.59$ & 0.17 & $0.00-0.36$ & 0.46 & $0.07-0.85$ & 0.63 & $0.13-1.13$ & & \\
\hline $9^{\circ}$ & 33 & 0.15 & $0.00-0.34$ & 0.55 & $0.24-0.86$ & 0.94 & $0.16-0.80$ & 0.24 & $0.05-0.43$ & & \\
\hline \multicolumn{12}{|l|}{ Age } \\
\hline$<20$ years of age & 15 & 0.00 & $0.00-0.00$ & 0.60 & $0.06-1.14$ & 0.40 & $0.00-0.94$ & 0.27 & $0.00-0.57$ & & \\
\hline 20 to 30 years of age & 133 & 0.18 & $0.08-0.28$ & 0.28 & $0.16-0.40$ & 0.38 & $0.24-0.52$ & 0.33 & $0.19-0.47$ & & \\
\hline \multicolumn{12}{|l|}{ Gender } \\
\hline Female & 84 & 0.10 & $0.03-0.17$ & 0.37 & $0.20-0.54$ & 0.43 & $0.25-0.61$ & 0.27 & $0.14-0.40$ & & \\
\hline Male & 64 & 0.25 & $0.08-0.42$ & 0.23 & $0.07-0.39$ & 0.31 & $0.11-0.51$ & 0.39 & $0.15-0.63$ & & \\
\hline
\end{tabular}




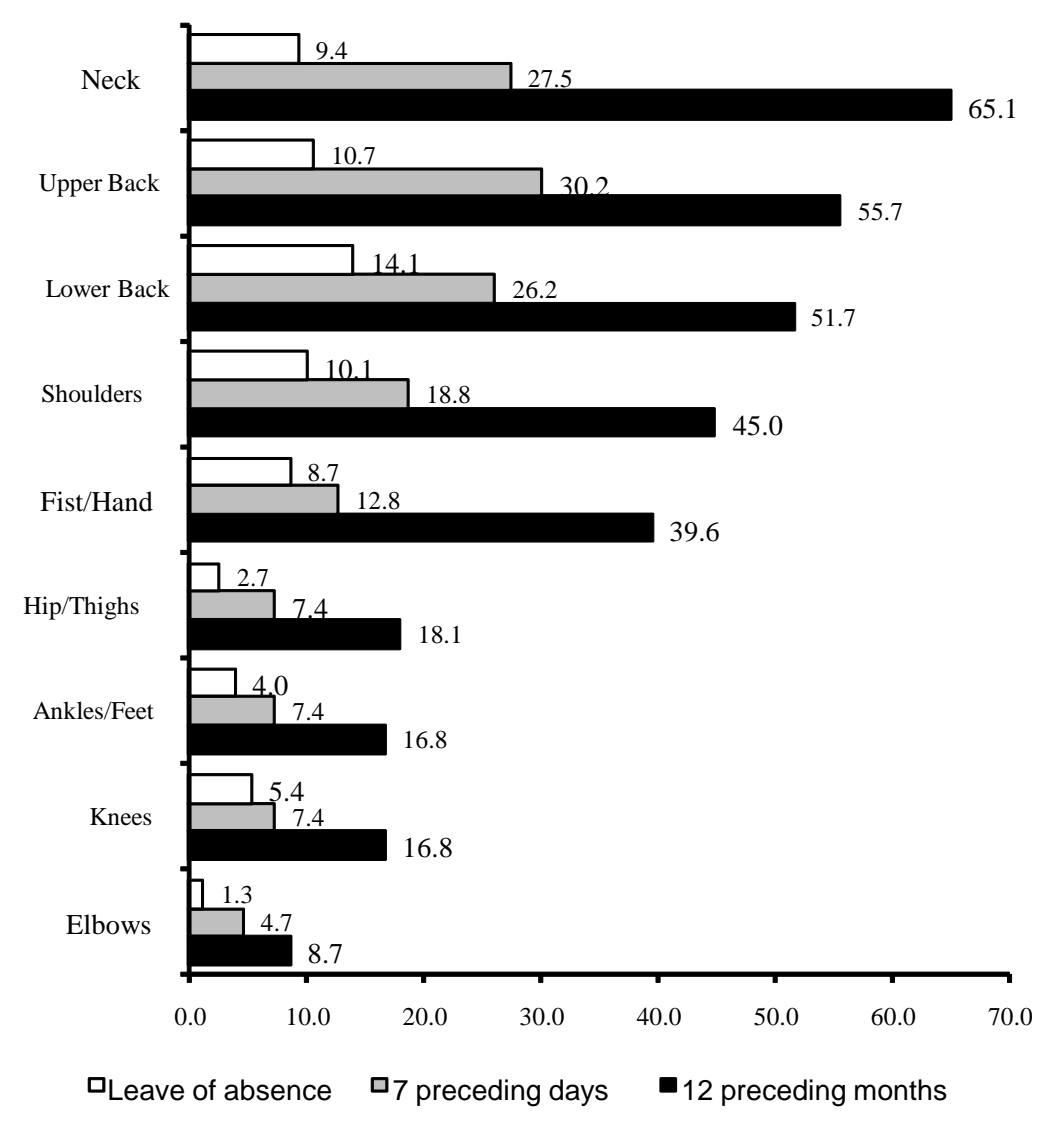

Figure 1. Frequency of symptoms and leave of absence by anatomic region in the students surveyed. Natal City, State of the Rio Grande do Norte, Brazil. 2013.

more than $50 \%$ of students, the most prevalent pain occurred in the neck, followed by pain in the upper back, lower back, shoulders, and fists/hands. Although neck pain was highlighted due its high prevalence, lower back pain (lumbar region) was responsible for the highest number of leave of absences.

\section{Discussions}

Data presented in Table 2 demonstrated an association between symptoms of musculoskeletal pain and length of time in the dental profession [8]-[10] [17]. For example, professionals who worked daily in dental clinics for more than 10 years without employing any preventive measures against work-related diseases (e.g., READ, DORT, hearing problems, etc.) developed musculoskeletal pain. Moreover, many professionals were forced to leave clinical practice early due to these health conditions, as demonstrated by the morbid events that increased progressively in relation to time spent in the clinic (Table 2). These results highlight the need for ergonomic principles to be implemented in the dental field to prevent occupational diseases. In addition, it is further advocated that teaching the concepts and benefits of ergonomics during the training of students in the dental field could greatly enhance the acknowledgment and implementation of healthy practices in future dental clinics [15] [17].

Previous studies [18]-[21] have mentioned the lack of adjustments in the workplace to accommodate ergonomic principles, including those relevant to the work environment and particularly the equipment. These decisions may result in the emergence of conditions or diseases affecting the musculoskeletal system, especially in the neck, shoulders, and the upper and lower back in different professional categories. These findings are in agreement with the results presented herein in Figure 1, which illustrated the painful symptomatology reported in different anatomical regions of undergraduate students from different academic levels. In addition, the percentages registered for the neck (65.1\%), upper (55.7\%) and lower (51.7\%) regions of the back, and shoulders (45.0\%), represent a worsening trend of symptoms. The symptoms reported herein were also consistent with 
those reported for the same anatomical regions in other studies [19] [20]. A statistically significant correlation was verified between the onset of these symptoms and time. For example, when symptoms occurred for a week and were not treated, symptoms continued for 12 months and eventually led to leave of absence. These results suggest that workers who start a professional life with a history of painful symptomatology present a high incidence of long-term musculoskeletal disorders [22] [23].

Regarding occupational diseases, it is important to remember that quality of life in the workplace should be a goal. Moreover, changes that improve quality of life benefit both the worker and the employer, with productivity longevity. A new model of health and safety routines for different occupational categories is evolving, and involves an expanded field of ergonomic options in work settings. Therefore, repositioning professionals and workers according to ergonomic principles is important, especially at the universities responsible for professional training. In the present study, the satisfaction of students regarding their knowledge of ergonomics was poor, with satisfaction levels ranging from $19.7 \%$ to $52.2 \%$ for students in different academic levels [24].

The determinants of posture in students, faculty, and staff of dental clinics in a Brazilian university were analyzed in [25]. The authors found no preventive measures in place, in addition to a lack of available ergonomic guidelines, therefore emphasizing the vital need for additional discussions on ergonomics and its application in academic settings. These results are similar to those obtained in the present study, where former students from the 5 th to 9 th semester reported averages of $43.8 \%, 86.1 \%, 95.7 \%$, 83.3\%, and $90.9 \%$ of students who did not perform preventive measures against occupational diseases. Turn et al. [26] also reported similar concerns with this issue, and advocated that ergonomic research be continued in order to improve the application of ergonomic principles and thereby improve the health and safety of workers.

For the majority of students surveyed on the concept of ergonomics, it was defined as professional attitudes and measures against diseases caused by repetitive strain. However, these concepts were not fully addressed or implemented during their academic study periods, thereby limiting the benefits that the implementation of ergonomic principles could provide [27].

According to the IEA, the definition of ergonomics has been consolidated since 1950, and represents a science that applies theories, principles, data, and methods to design environments and to optimize human wellbeing and performance within a system in general. Furthermore, in the United States, ergonomics additionally represents a scientific area related to understanding interactions between humans and other elements (e.g., environment, equipment, instruments) [27].

Regarding Table 2 and Table 3, the results are similar to those of Soriano et al. [27] who investigated dental students at a university in Pernambuco, Brazil and reported the percentage of students experiencing musculoskeletal pain according to gender. In that study, 61.3\% of female students were affected. Similarly, female students were more affected by musculoskeletal disorders in the present study, especially in the neck. Other authors [11] have justified gender bias by indicating that females present a reduced capacity to store glycogen into usable energy, fewer muscle fibers, lower bone density and smaller bone size, and the stages of pregnancy and menopause can further affect susceptibility to musculoskeletal disorders.

Different methods for reporting symptoms of pain have previously been compared [4] [16] with the aim of assessing content validity, psychometric properties, feasibility of use, and reliability. In each study, the instruments were found to be feasible, although advantages and disadvantages were associated with applicability. In the present study, a validated Brazilian version of the Nordic Musculoskeletal Questionnaire [16] was used and translated into several languages. The Brazilian version was developed to standardize the measurement of reported musculoskeletal symptoms to identify the presence of disorders. The lack of standardization in the classification of musculoskeletal injuries remains a worldwide challenge in the field of epidemiology, particularly regarding the diagnosis and treatment of work-related injuries [6].

There is a clear need to adopt, implement, and supervise habits, work postures, breaks and stretches throughout clinical care. These measures must be urgently implemented in workplaces that require a higher activity of the musculoskeletal system [18]. Based on the philosophy of quality and safety for healthcare professionals, the authors suggest a protocol for the prevention of diseases in the university environment, the Pergodonto Protocol (ergonomics in dentistry). The acceptance and implementation of this protocol would be as an important initiative for the adoption of basic ergonomic guidelines, thereby preventing occupational diseases. Implementation of these guidelines would be especially relevant to address the symptoms of pain already reported by students during training processes. 


\section{Conclusion}

Regardless of academic level, the concept of ergonomics expressed by the surveyed students was not consistent with the definition established by the IEA. Furthermore, pain symptoms were reported more often by female dental students, and the anatomical region most affected was the neck, followed by the lower back, wrists, hands, and shoulders. The results of the survey also demonstrated that pain was reported in these regions over the previous 12 months or 7 days preceding the survey. It is therefore essential and urgent that regulatory measures or procedures are employed to aid in the prevention of musculoskeletal diseases in the university environment. It is unacceptable that dental students undergoing training already present musculoskeletal conditions, especially with the level of statistical significance observed.

\section{Suggestions}

For students undergoing training in the dental profession that already experience a high frequency of pain symptoms, the implementation of ergonomic practices in educational institutions and preventive dental clinics would represent an important improvement. Considering the vulnerability of the posture of the student, and the complex systems of interaction between students and the university environment and equipment, there are several opportunities to introduce ergonomic principles. For UFRN, a specific action that should not be ignored is the opportunity to implement a protocol to address ergonomic considerations as a part of the routine procedure of the institutional biosafety protocol already accepted at most universities in Brazil. Ergonomic considerations refer to those related to adequate work postures, satisfactory levels of workplace lighting, instruments and equipment within healthcare work regulations, utilization of odontology assistants, avoidance of osteomuscular burden, and increase of productivity in an optimized manner, with breaks between patients. The recommended protocol, called the PERGODONTO Protocol (Ergonomics in Dentistry), consists of the following steps:

-Professional standards and ergonomic guidelines are posted in dental clinics so that supervision and compliance with rules are carried out daily.

-Daily sessions of stretching prior to clinical care are conducted in accordance with the planning of clinical discipline curriculum [27].

-Guidelines and postural adjustments are followed by students according to the regulation (NR17) of the Brazilian Ministry of Labor [27].

\section{Limitations of the Study}

The study presents a few limitations, such as: it was not considered whether the interviewees presented any worsening related to osteomuscular pain before dentistry professional practice, if regular physical exercises were practiced to prevent osteomuscular disorders, or if any of the participants had ever undergone surgical procedures to correct motor system disorders, such as disk protusion. The study presented herein can serve as a basis for a retrospective cohort, directed to monitoring, especially of those who presented recurrent painful symptomatology.

\section{References}

[1] Verhagen, A.P., Karels, C., Bierma-Zeinstra, S.M.A., Feleus, A., Dahaghin, S. and Burdorf, A. (2007) Exercises Proves Effective in a Systematic Review of Work-Related Complaints of the Arm, Neck, or Shoulder. Journal of Clinical Epidemiology, 60, 110-117. http://dx.doi.org/10.1016/j.jclinepi.2006.05.006

[2] Regis Filho, G.I., Zmijevski, T.R.L., Pietrobon, L., Fadel, M.A.V. and Klug, F.K. (2010) Occupational Exposure of Dentists to Mechanical Vibration Transmitted through the Hands: A Case Study. Revista Produção, 20, 502-509.

[3] Brazil. Social Security. National Institute of Social Security (2003) Normative INSS/DC No 98, December 5th of 2003. Approves Technical Standard on Repetitive Strain Injury—RSI or Work-Related Musculoskeletal Disorders—WMSD. Diário Oficial União, Brasília, Seção 1.

[4] Jolles, B.M., Buchbinder, R. and Beaton, D.E. (2005) A Study Compared Nine Patient-Specific Indices for Musculoskeletal Disorders. Journal of Clinical Epidemiology, 58, 791-801. http://dx.doi.org/10.1016/j.jclinepi.2005.01.012

[5] Eerd, D.V., Beaton, D., Cole, D., Lucas, J., Hogg-Johnson, S. and Bombardier, C. (2003) Classification System for Upper-Limb Musculoskeletal Disorders in Workers: A Review of Literature. Journal of Clinical Epidemiology, 56, 925-936. http://dx.doi.org/10.1016/S0895-4356(03)00122-7 
[6] Régis Filho, G.I., Michels, G. and Sell, I. (2009) Repetitive Strain Injury Work-Related Musculoskeletal Disorders of Dentists: Biomechanical Aspects. Revista Produção, 19, 569-580.

[7] Carneiro, P.M.S. (2005) Ergonomic Analysis of Posture and Movements in the Profession of Dentist [Dissertation Online]. University of Minho, Portugal. http://hdle.net/1822/960

[8] Kotliarenko, A., Michel-Crosato, E., Biazevic, M.G.H., Crosato, E. and Silva, P.R. (2009) Musculoskeletal Disorders and Associated Factors among Dentists Midwestern State of Santa Catarina. Revista Odonto Ciência, 24, 173-179.

[9] Regis Filho, G.I., Michels, G. and Sell, I. (2006) Repetitive Strain Injury Work-Related Musculoskeletal Disorders in Dentists. Revista Brasileira de Epidemiologia, 9, 346-359.

[10] Teles, C.J.C.F. (2009) Assessing the Degree of Knowledge of Physicians-Dentists Regarding the Application of Ergonomics in Dentistry [Monograph]. Universidade Fernando Pessoa, Porto.

[11] Valério, S.B., Lima, J.S. and Oliveira, A.M.K. (2008) Prevalence of Pain in the Spine of Dentists in Dourados-MS. Revista Interbio, 2, 56-57.

[12] Lida, I. (2005) Ergonomics: Projetc and Prodution. 2nd Edition, Edgar Blucher, São Paulo.

[13] Brazil (2001) Ministry of Health, Department of Health Policy and Programmatic Actions Departments of Strategic. Technical Area of Occupational Health. Repetitive Strain Injuries (RSI), Work-Related Musculoskeletal Disorders (MSDs). Ministry of Health, Brasilia.

[14] Pinheiro, F.A., Amaral, B.T. and Carvalho, C.V. (2002) Validation of Questionnaire Nordic Musculoskeletal Symptoms as a Measure of Morbidity. Revista de Saúde Pública, 36, 307-312. http://dx.doi.org/10.1590/S0034-89102002000300008

[15] Jesus, L.F., Marinha, M.S. and Moreira, M.F.R. (2010) Musculoskeletal Disorders in Dentists: A Literature Review. Revista Uniandrade, 11, 75-87. http://dx.doi.org/10.18024/1519-5694/revuniandrade.v11n1p75-88

[16] Pillastrini, P., Mugnai, R., Bertozzi, L., Costi, S., Curti, S., Guccione, A., et al. (2010) Effectiveness of an Ergonomic Intervention on Work-Related Posture and Low Back Pain in Video Display Terminal Operators: A 3 Year Cross-Over Trial. Applied Ergonomics, 41, 436-443. http://dx.doi.org/10.1016/j.apergo.2009.09.008

[17] Wichansky, A.M. (2000) Usability Testing in 2000 and Beyond. Ergonomics, 43, 998-1006. http://dx.doi.org/10.1080/001401300409170

[18] Pentikis, J., Lopez, M. and Thomas, R. (2002) Ergonomic Evaluation of a Government Office Building. Work, 18, 123131.

[19] Pilligan, G., Herbet, R., Hearns, M., Dropkin, J., Landsbergis, P. and Cherniack, M. (2000) Evaluation and Management of Chronic Work-Related Musculoskeletal Disorders of the Distal Upper Extremity. American Journal of Industrial Medicine, 37, 75-93. http://dx.doi.org/10.1002/(SICI)1097-0274(200001)37:1<75::AID-AJIM7>3.0.CO;2-4

[20] Michel-Crosato, E., Calvielli, T.P., Biazevic, M.G.H. and Crosato, E. (2003) Socioeconomic Profile of the Workforce Represented by Graduates FOUSP (1990-1998). RPG—Revista da Pós-Graduação, 10, 217-226.

[21] Graça, C.C., Araújo, T.M. and Silva, C.E.P. (2006) Factors Associated with the Prevalence of Self-Reported Musculoskeletal Pain in Dentists. Revista ABO Nacional, 14, 225-231.

[22] Dul, J. and Neumann, W.P. (2009) Ergonomics Contributions to Company Strategies. Applied Ergonomics, 40, 745752. http://dx.doi.org/10.1016/j.apergo.2008.07.001

[23] Martins, M.S.A. (2008) Ergonomics and Dentistry: Determinants of Body Posture Biomechanical Risk Students the School Clinic for a Federal University. Dissertation, Universidade Federal do Rio Grande do Norte, Natal (RN).

[24] Saarni, S.I., Suvisaari, J., Sintonen, H., Koskinen, S., Harkanen, T. and Lonnqvist, J. (2007) The Health-Related Quality-of-Life Impact of Chronic Conditions Varied with Age in General Population. Journal of Clinical Epidemiology, 60, 1288-1297. http://dx.doi.org/10.1016/j.jclinepi.2007.03.004

[25] Garbin, A.J.I., Garbin, C.A.S. and Diniz, D.G. (2009) Standards and Ergonomic Guidelines in Dentistry: The Path to Adopting a Healthy Working Posture. Revista de Odontologia da Universidade de São Paulo, 21, 155-161.

[26] Tulder, M.V., Malmivaara, A. and Koes, B. (2007) Repetitive Strain Injury. The Lancet, 369, 1815-1822. http://dx.doi.org/10.1016/S0140-6736(07)60820-4

[27] Soriano, E.P., Carvalho, M.V.D., Caldas Júnior, A.F., Coelho Junior, L.G.T.M., Vidal, H.G. and Araujo, A.B.V.L. (2009) From Theory to Practice: Pain Related to MSDs and Ergonomics Knowledge among Dental Students. Brazilian Oral Research, 23, 160. 


\section{Appendix. Nordic Musculoskeletal Questionnaire}

MUSCULOSKELETAL DISORDERS

Please answer by putting a cross in the appropriate box one cross for each question. Please answer every question even if you have never had trouble in any parts of your body. This picture shows how the body has been divided. You should decide for yourself which part (if any) is or has been affected.

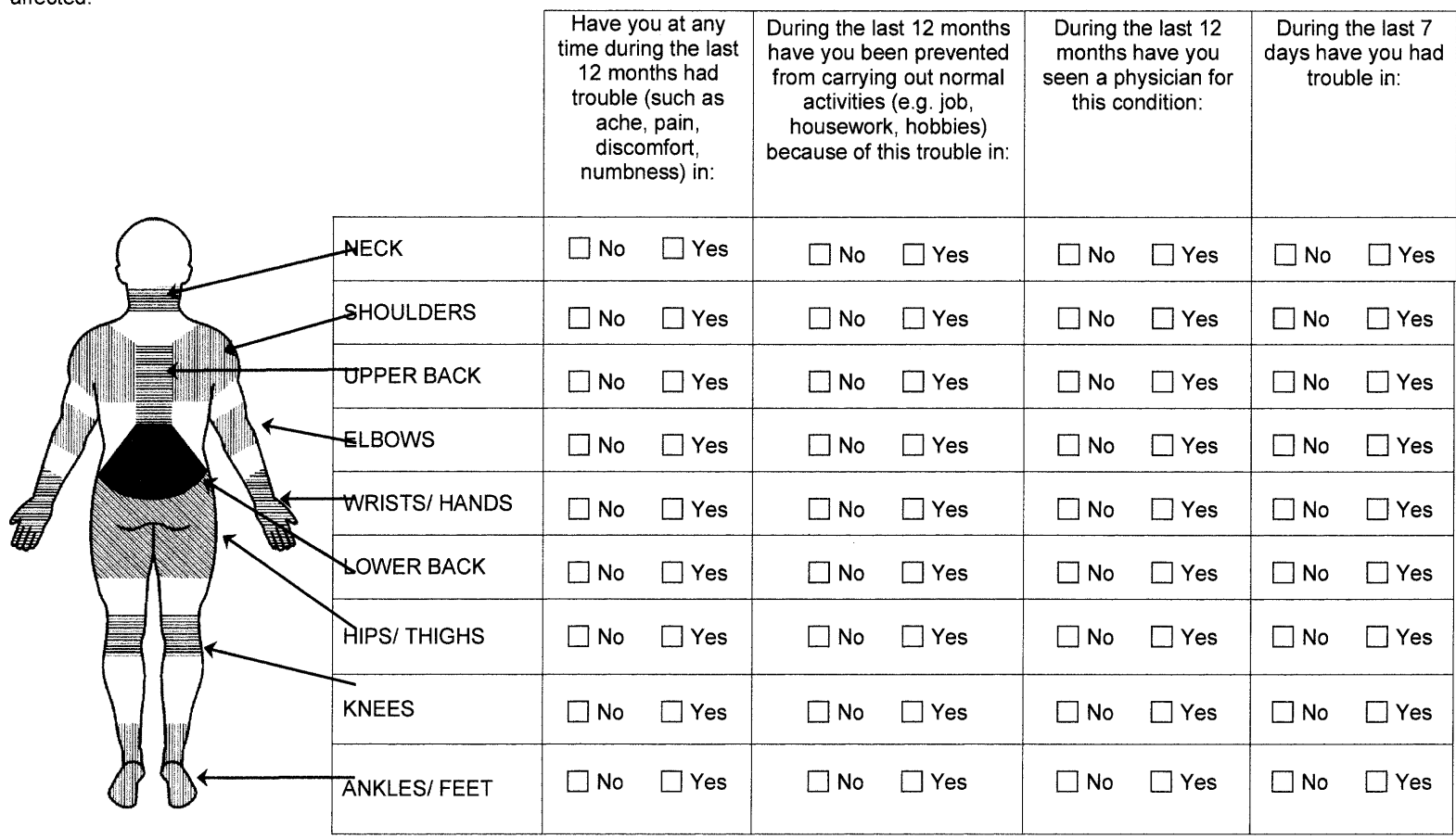

